\title{
Optimal Moving Grids for Time-Dependent Partial Differential Equations
}

\author{
A.J. Wathen
}

September 1989

$1 N-64$

DAL ONLARIAC 43036 Research Institute for Advanced Computer Science
NASA Ames Research Center

RIACS Technical Report 89.42

NASA Cooperative Agreement Number NCC2-387

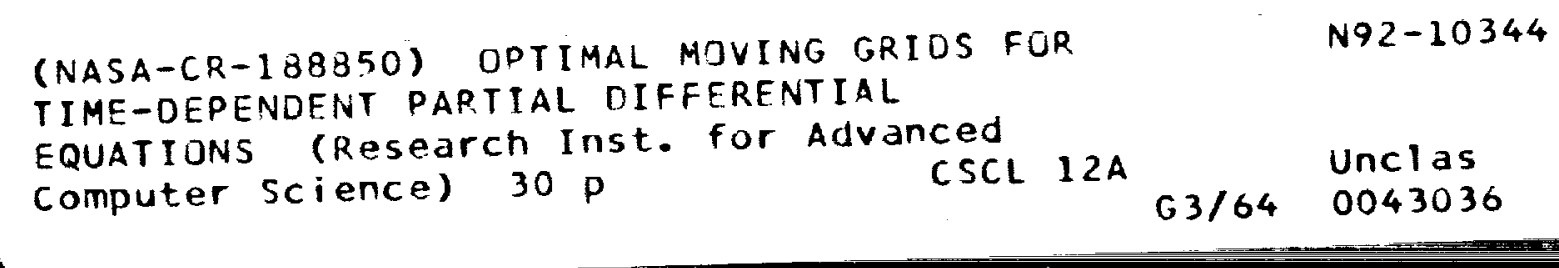




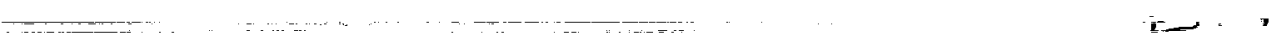




\title{
Optimal Moving Grids for Time-Dependent Partial Differential Equations
}

\author{
A. J. Wathent \\ Research Institute for Advanced Computer Science \\ NASA Ames Research Center \\ RIACS Technical Report 89.42 \\ September 1989
}

\begin{abstract}
Various adaptive moving grid techniques for the numerical solution of time-dependent partial differential equations have been proposed. The precise criterion for grid motion varies, but most techniques will attempt to give grids on which the solution of the partial differential equation can be well represented. We investigate moving grids on which the solutions of the linear heat conduction and viscous Burgers' equation in one space dimension are optimally approximated. Precisely, we report the results of numerical calculations of optimal moving grids for piecewise linear finite element approximation of PDE solutions in the least squares norm.
\end{abstract}

†School of Mathematics, University of Bristol, University of Walk, Bristol BS8 1TW, England and Visiting Research Scientist at the Research Institute for Advanced Computer Science. Work reported herein supported by Cooperative Agreement NCC2-387 between the National Aeronautics and Space Administration (NASA) and the Universities Space Research Association (USRA). 


\section{Introduction}

For many time-dependent Partial Differential Equations, particularly those which admit solutions with evolving sharp features such as fronts, use of moving grids can be an extremely useful tool for obtaining accurate numerical solutions efficiently. A number of moving grid procedures have been suggested for different problem classes ([1], [16], [18], [9], [21], [11])

Grid adaptive strategies for time-dependent partial differential equations essentially fall into one of two categories: those where grid adaptation is carried out at discrete time levels, and those where the adaptive process is performed continuously in time. In some instances this distinction is weak since a discrete time adaptive strategy can sometimes be regarded as a discretisation of an underlying differential equation which describes grid motion (Coyle, Flaherty \& Ludwig [7]). Coyle et al. ([7]) give examples where such Ordinary Differetial Equations's describing the moving grid are nonlinearly unstable even for simple smooth problems such as the equation of linear heat conduction

$$
u_{t}=\sigma u_{x z}
$$

so that very highly oscillatory grid motions result.

These studies have important bearing on the implimentation of adaptive gridding strategies in Method of Lines software ([15], [4], [20], [19], [13]) where Ordinary Differential Equation (or Differential Algebraic Equation) solvers are used to solve the Ordinary Differential Equations resulting from spatial discretisation. A rapid grid motion will certainly introduce stiffness if the PDE solution is evolving on a slower time scale.

In the Moving Finite Element Method of Miller ([16], [6], [10]) (see also [17], [12], [3]) and the work of Adjerid \& Flaherty [1], [2], the grid motion equations are solved simultaneously with the equations which describe the PDE solution on the moving grid. In such situations, the smoothness in time of the spatial discretisation is certainly an important factor.

In this paper we describe the results of a series of numerical experiments which are designed to examine how good a solution one can hope to achieve by continuously adapting the computational grid. The experimental set-up is simple: piecewise linear finite elements (linear splines) on a closed interval in one dimension. The two boundary nodes are fixed, and the number of free internal nodes is not varied. Precisely we investigate the problem of computing the optimal grid node positions of the best least squares free knot linear spline approximation to the spatial solution of a PDE at different times. The loci of such optimal positions define an optimal trajectory for 
each node. The ensemble of these nodal trajectories defines the optimal moving grid in this norm. Piecewise linear representation is used here simply because it is a common choice. The choice of least squares norm is more arbitrary, however the results of Cullen \& Morton [8] (see also [9], [3]) indicate that this may be an appropriate norm at least for the finite element method. The results of Carey \& Dinh [5] show that the free knot linear spline interpolation problem in the least squares norm is approximately equivalent to equidistribution of the monitor function $\left(f^{n}\right)^{(2 / 5)}$.

The problem of free knot spline approximation is nonlinear and great care is required to isolate the global minimum from potentially many locally optimal fits. In the work reported here, we have used a code based on the hybrid technique described by Loach and Wathen [14] for the approximation problem. Though no proof of global convergence exists for this algorithm, there is considerable evidence that the solutions it produces are indeed globally optimal. The testing of this routine used considerably more complicated test functions than the solutions of simple PDE's presented here.

The planned comparison with proposed adaptive strategies became irrelevant when the highly non-smooth character of the optimal grid node positions was observed even for the two very simple problems that were used in this study.

We should emphasise that the computation of the optimal approximation is an expensive procedure, and relies on exact knowledge of the PDE solution. The numerical techniques that we use here are therefore not at all suitable as practical moving grid methods for partial differential equations.

In section 2 we describe the experiment and we show sample results in section 3. Section 4 covers our deductions and conclusions.

\section{Optimal Grid Experiment}

It was desired to consider both parabolic and hyperbolic problems, thus two test problems were used.

The first was the parabolic problem of linear heat conduction

$$
u_{t}=u_{x x}, \quad 0 \leq x \leq 1, t \geq 0
$$


with homogeneous Neumann boundary conditions, $u_{n}=0$ (here $n$ is the normal direction to the boundary). In order that there be a rapidly computable analytic solution, initial data with few Fourier modes is employed. If a single mode is used, the PDE solution is simply an exponential decay of this original mode. The scale-invariance of the $l_{2}$ best fit equations then implies that the nodal positions in the optimal grid are fixed independently of time. The initial data is thus chosen as

$$
u(x, t=0)=\cos \pi x+\cos 2 \pi x
$$

so that the analytic solution is simply

$$
u(x, t)=e^{-\pi^{2} t} \cos \pi x+e^{-4 x^{2} t} \cos 2 \pi x .
$$

For every instant in time, $t$, this describes the function to be approximated by the optimal free knot linear spline in space,$x$.

The second problem is the viscous Burgers' equation

$$
u_{t}+u u_{x}=\epsilon u_{x x}, \quad 0 \leq x \leq 1, t \geq 0
$$

which is nearly hyperbolic for small values of $\epsilon$. A particular analytic solution of this problem can be found using the Cole-Hopf transformation giving

$$
u(x, t)=f(\psi)
$$

where

$$
\psi=x-\mu t-\beta
$$

and

$$
f(\psi)=\left[\mu+\alpha+(\mu-\alpha) e^{(\alpha \psi / \alpha)}\right] /\left[1+e^{(\alpha \psi / \alpha)}\right] .
$$

$\epsilon$ is taken as 0.01 , and the arbitrary constants $\dot{\alpha}, \beta$ and $\mu$ are chosen to be $0.4,0.125$ and 0.6 respectively so that the solution is a right-ward moving steep front as $t$ increases. The boundary conditions are time-dependent Dirichlet conditions taken from this exact solution.

For the near hyperbolic problem, the characteristics of the corresponding purely hyperbolic problem $u_{t}+u u_{z}=0$ are likely to be near the optimal trajectories in this approximation experiment because of the advective nature of the solution. However, for the parabolic heat flow equation, no a priori 'good' node trajectories are apparent. 
The approximation problem is solved with fixed knots at the boundary points 0 and 1 , but free spline values at these end points. That is, if $x_{0}=0, x_{n+1}=1$ are fixed knots and $x_{1}, x_{2}, \ldots, x_{n}$ are free knots, and $a_{i}$ is the value taken by the linear spline $S$ at $x_{i}$ for $i=0,1, \ldots n+1$, then

the approximation problem is to minimise $\|f-S\|_{l_{2}[0,1]}$ with respect to $x_{i}, i=1,2, \ldots, n$ and $a_{i}, i=0,1, \ldots, n+1$, where $f$ is the function to be approximated.

\section{Computational Results}

We describe first the results for the linear heat flow equation.

The simplest case we have considered is when the piecewise linear approximating function has only four (free) knot points. We present four figures to display the results.

Figure 3.1 shows the loci of the optimal nodal trajectories, the analytic solution at selected times, and the best free knot spline approximants to the analytic solution at these instants, all plotted in the u-x plane. That is, we have plotted the analytic solution (dotted line) and its best least squares fit (dashed line) at a number of discrete times, and at many smaller time intervals have plotted the optimal location in $u-x$ space of the optimal node position. These latter are joined by the solid line to indicate the loci of the node points which define the optimal grid.

One immediate observation is that the optimal node point trajectories undergo a discontinuous jump at two particular instances $t \simeq 0.007$ and $t \simeq 0.058$ during $0 \leq t \leq 0.91$ which is the time interval chosen for the experiment. Figure 3.2 shows only the nodal trajectories in u-x space, and figure 3.3 shows these same trajectories when projected onto the $x-t$ plane. The qualitative similarity of this last representation with the results presented in Adjerid \& Flaherty ([2]) is striking. The $x-t$ trajectories of nodes in an adaptive grid presented in Adjerid \& Flaherty appear to move smoothly at times and then to veer sharply. There appears a close analogy between this veering and the precisely discontinuous nature of the optimal grid. Figure 3.4 shows the least squares error of the optimal linear spline. It is continuous as is expected, but appears to have a discontinuous derivative at the two instances when the node positions jump. 
We show the results of one other experiment on the same problem but with ten free nodes. We present the results graphically as above in figs 3.5, 3.6, 3.7 and 3.8. We are again able to clearly see discontinuous motion of the optimal nodal trajectories, though they are apparently piecewise smooth. The $l_{2}$ error is again continuous in time.

Several other experiments were performed on this heat flow problem with qualitatively similar results. We comment that there appear to be no 'boundary effects' caused by our choice of end conditions for this test problem.

Several experiments for the Viscous Burgers Equation were performed. We present only the results for 2, 3 and 6 free nodes. The graphical results are presented in the same way as for the heat flow equation

For the 2 node solution the optimal nodal trajectories are actually smooth as shown in figs. 3.9, 3.10 and 3.11. They are almost exactly characteristic trajectories for, the corresponding hyperbolic problem $(\epsilon=0)$ though they are slightly curved. The least squares error is smooth (fig. 3.12) from $t=0$ to $t=1.16$ which is the period of the experiment.

When 3 free nodes are used, figures $3.13,3.14$ and 3.15 show however that the optimal nodal trajectories are again discontinuous. The least squares error (fig. 3.16) is continuous but, as before, has a 'corner'. This problem is also run from $t=0$ to $t=1.16$.

For our final experiment we have run from $t=-0.23$, when the steep front is just moving into the domain, until $t=1.21$. In this case we used 8 free nodes. The results plotted in figures 3.17, 3.18, 3.19 and 3.20 show the discontinuous movement of the nodes as the front enters the domain followed by smooth characteristic-like motion as the front moves across the domain. The $l_{2}$ error is again seen to be continuous. We see in this example the effect of the solution entering through the domain boundary. Even for the corresponding hyperbolic inviscid Burgers' equation, a boundary condition at this left hand boundary would be required as the characteristics enter the domain here. 


\section{Conclusions}

We have computed the optimal nodal trajectories of best free knot spline approximations to the solutions of two one-dimensional test problems. That is, we have computed the moving grids on which the analytic solution of the partial differential equation can be best represented at every instant of time by a linear spline with a fixed number of nodes.

We find that the optimal nodal trajectories are discontinuous for both of our test functions - the parabolic linear heat conduction equation and the nonlinear convection-dominated viscous Burgers' equation. In the latter case the optimal paths are similar to characteristic trajectories of the corresponding hyperbolic equation (the Inviscid Burgers' equation) when the solution is not significantly influenced by the boundaries.

For more complicated time-dependent partial differential equation problems, we must expect to obtain similar behaviour in general.

We conclude that global optimal approximation continuously in time is not a useful property to try to achieve with a moving grid strategy because of the difficulties in trying to time integrate discontinuous optimal nodal paths. Regularisation may be a remedy in some situations. There may be smooth paths associated with local optima, but there must be expected to be locally optimal grids on which the error is large. However, following a smooth locally optimal grid may be useful in some problems.

\section{Acknowledgements}

This work was carried out whilst the author was at RIACS at NASA Ames. I am grateful to Richard Sincovec and Neil Madsen for useful discussions on this work.

\section{References}

[1] S. Adjerid \& J. E. Flaherty, A Moving finite element Method with Error Estimation and Refinement for one-dimensional time-dependent partial differential equations, SIAM J. Numer. Anal. 23, 778-796, 1986.

[2] S. Adjerid \& J. E. Flaherty, A Moving-Mesh Finite Element Method with Local Refinement for Parabolic Partial Differential Equations, Comput. Meths. Appl. Mech. Engrg. 55, 3-26, 1986. 
[3] M. J. Baines \& A. J. Wathen, Moving Finite Elements for evolutionary problems I: Theory, J. Comput. Phys. 79(2), 245-269, 1988.

[4] M. Berzins \& R. M. Furzeland, A User's Manual for SPRINT - A Versatile Software Package for Solving Systems of Algebraic, Ordinary and Partial Differential Equations: Part 2 - Solving Partial Differential Equations, Report No. 202, Department of Computer Studies, The University of Leeds, UK, 1986.

[5] G. F. Carey \& H. T. Dinh, Grading Functions and Mesh Redistribution, SIAM J. Numer. Anal. 22, 1028-1040, 1985.

[6] N. Carlson \& K. Miller, Gradient Weighted Moving Finite Elements in Two Dimensions, Report PAM-342, Center for Pure and Applied Mathematics, University of California, Berkeley, September 1986.

[7] J. M. Coyle, J. E. Flaherty \& R. Ludwig, On the Stability of Mesh Equidistribution Strategies for Time-Dependent Partial Differential Equations, J. Comput. Phys. 62(1), 26-39, 1986.

[8] M. J. D. Cullen \& K. W. Morton, Analysis of Evolutionary Error in Finite Element and Other Methods, J. Comput. Phys. 34, 245-268, 1980.

[9] S. F. Davis \& J. E. Flaherty, An Adaptive Finite Element Method for Initial-Boundary Value Problems for Partial Differential Equations, SIAM J. Sci. Stat. Comput. 3(1), 6-27, 1982.

[10] J.Djomehri \& K. Miller, A Moving Finite Element Code for General Systems of PDE's in 2-D, Report PAM-57, Center for Pure and Applied Mathematics, University of California, Berkeley, October 1981.

[11] A. Harten \& J. M. Hyman A Self-adjusting Grid for the Computation of Weak Solutions of Byperbolic Conservation Laws, J. Comput. Phys. 50, 235-269, 1983.

[12] B. M. Herbst, S. W Schoombie \& A. R. Mitchell, Equidistributing Principles in Moving Finite Element Methods, J. Comput. \& Appl. Math. 9, 377-389, 1985.

[13] A. C. Hindmarsh, ODE Solvers for use with the Method of Lines, pp 312-316 of Advances in Computer Methods for partial differential EquationsIV, R. Vichnevetsky \& R. S. Stepleman ,eds. , IMACS, 1981.

[14] P. D.Loach \& A. J. Wathen, On the Best Least Squares Approximation of Continuous Functions using Linear Splines with Free Knots, Report AM-88-14, School of Mathematics, University of Bristol, September 1988. IMA J. Numer Anal. to appear. 
[15] N. K. Madsen \& R. F. Sincovec, The Numerical Method Lines, Computational Methods in Nonlinear Mechanics, J. T. Oden et al., eds. , TICOM, Austin, Texas, 1974.

[16] K. Miller \& R. N. Miller, Moving Finite Elements I, SLAM J. Numer. Anal. 18, 1019-1032, 1981. \& K. Miller, Moving Finite Elements II, SIAM J. Numer. Anal. 18, 1033-1057, 1981.

[17] A. C. Mueller \& G. F. Carey, Continuously Deforming Finite Elements, Int. J. Numer. Meths. Engrg. 21, 2099-2126, 1985.

[18] L. R. Petzold, An Adaptive Moving Grid Method for One-dimensional Systems of Partial Differential Equations and its Numerical Solution, UCRL100289 Preprint (Lawrence Livermore National Laboratory), October 1988.

[19] L. R. Petzold, A Description of DASSL: A Differential/Algebraic System Solver, IMACS Trans. on Scientific Computation, Vol 1, R. S. Stepleman, ed. , 1982.

[20] R. M. Furzeland, J. G. Verwer \& P. A. Zegeling, A Numerical Study of three Moving Grid Methods for One-Dimensional Partial Differential Equations which are based on the Method of Lines, Report NM-R8806, Department of Numerical Mathematics, CWI, Amsterdam, June 1988.

[21] J. G. Verwer, J. G. Blom \& J. M. Sanz-Serna, An Adaptive Moving Grid Method for One-Dimensional Systems of Partial Differential Equations, J..Comput. Phys. 82, 454-486, 1989. 
Optimal Nodal Trajectories

Linear Heat Flow Equation

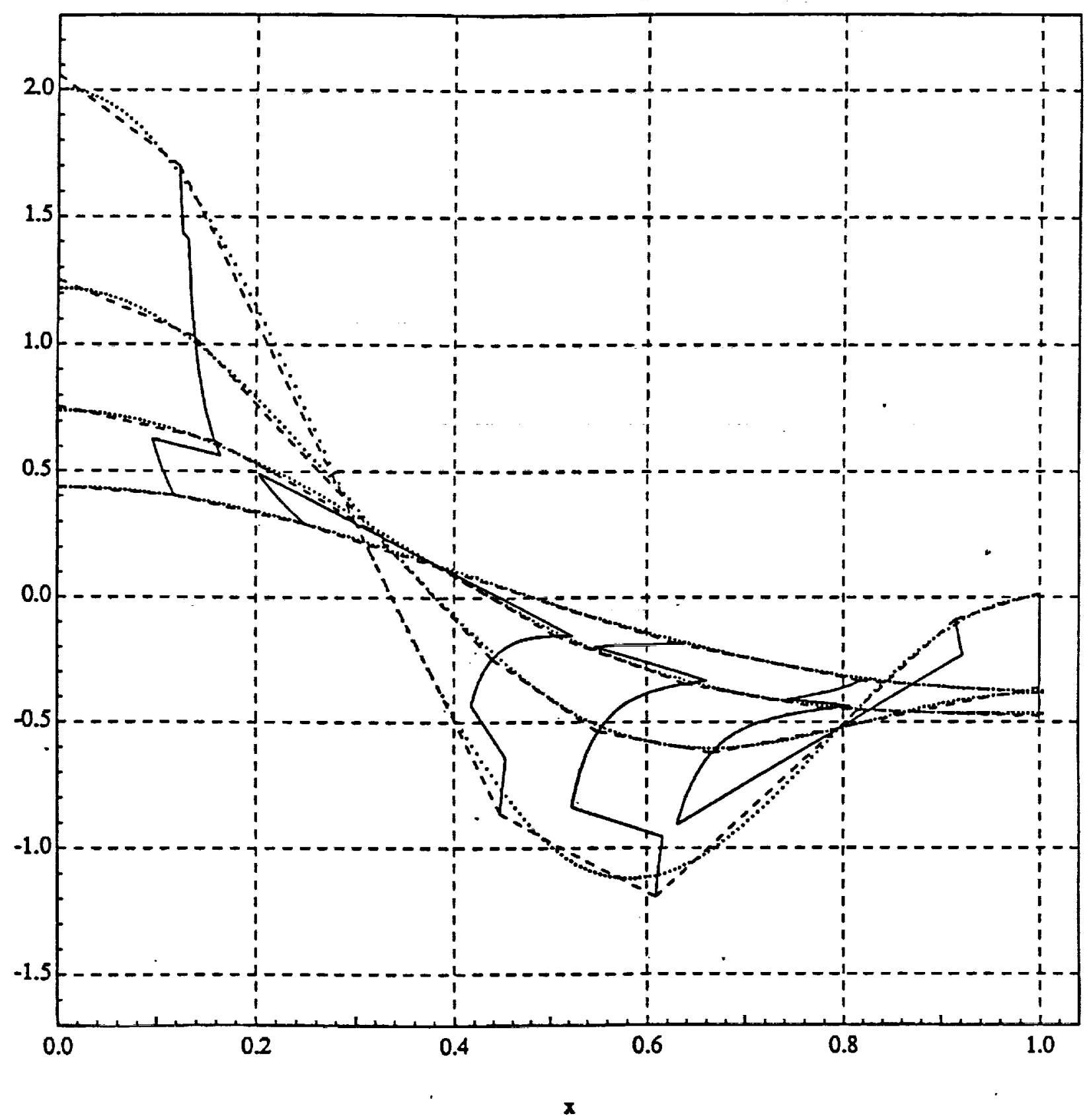

Figure 3.1. Linear Heat Equation with 4 free nodes: exact solution (dotted line), best fits (dashed line) and optimal nodal trajectories (full line) all in $u-x$ space. 
Optimal Nodal Trajectories

\section{Linear Heat Flow Equation}

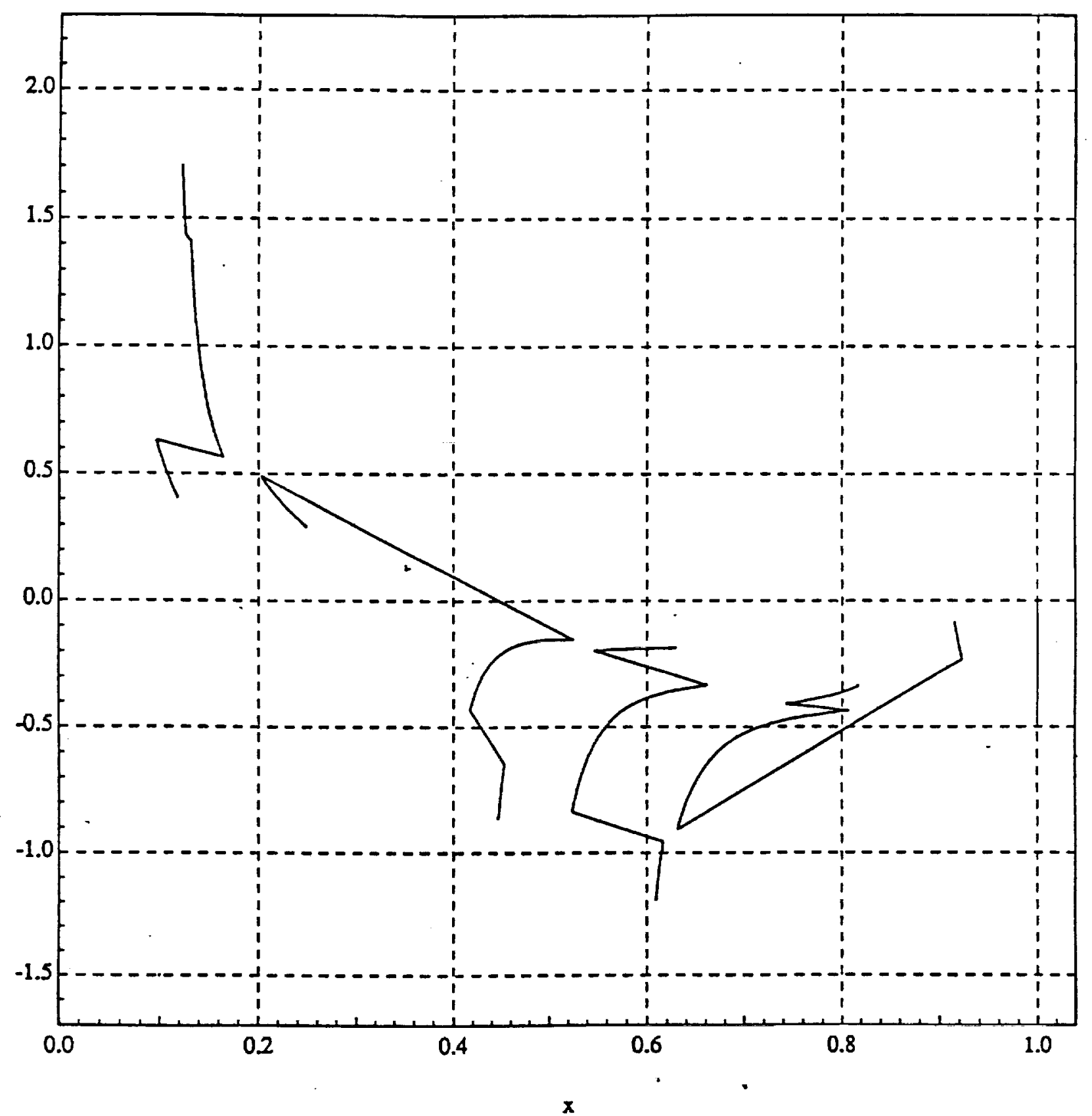

Figure 3.2. Linear Heat Equation with 4 free nodes: optimal nodal trajectories in $u-x$ space. 
Optimal Nodal Trajectories in $x-t$ space

Linear Heat Flow Equation

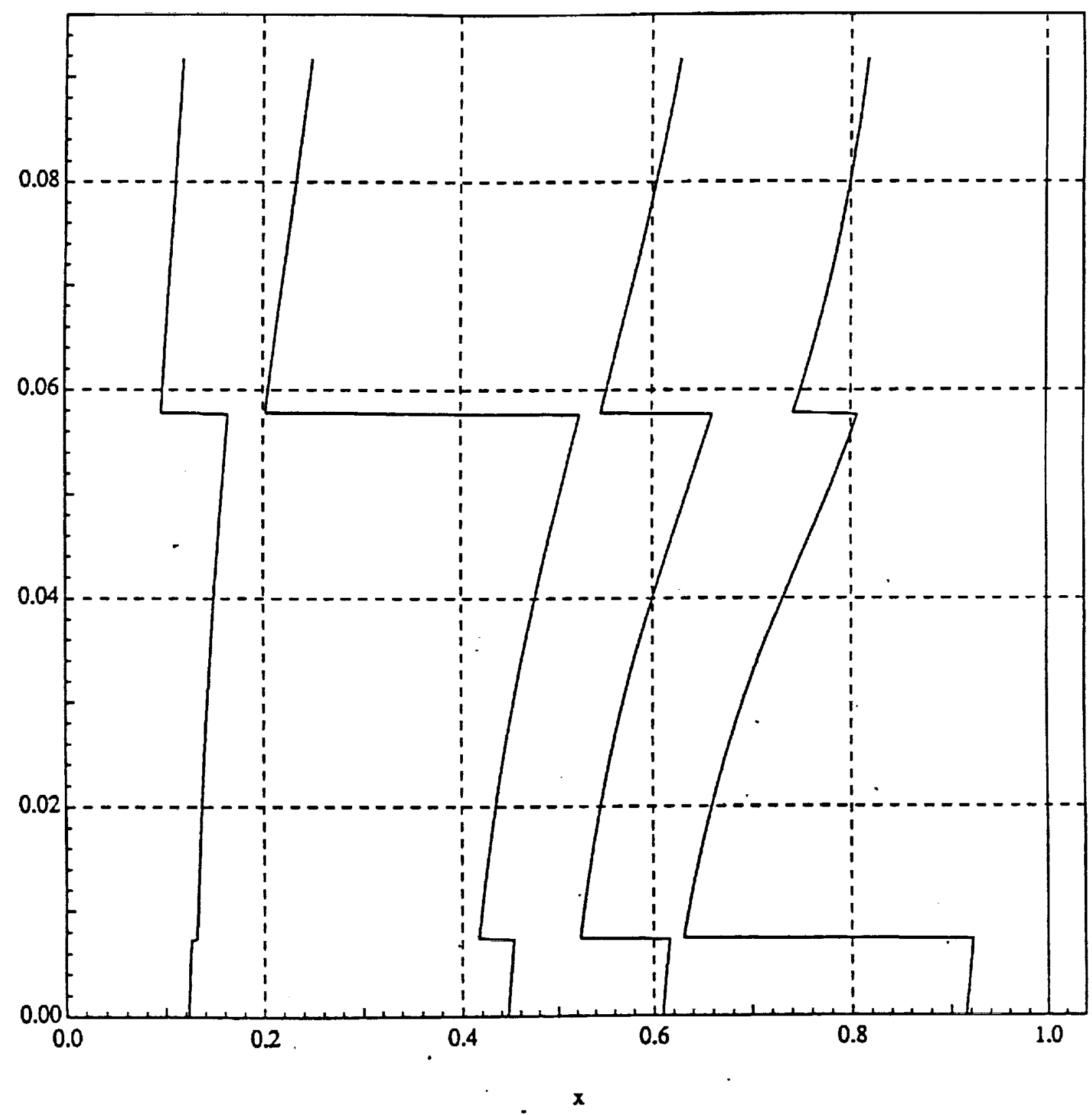

Figure 3.3. Linear Heat Equation with 4 free nodes: optimal nodal trajectories in $x$-t space. 
Least Squares Error

Linear Heat Flow Equation

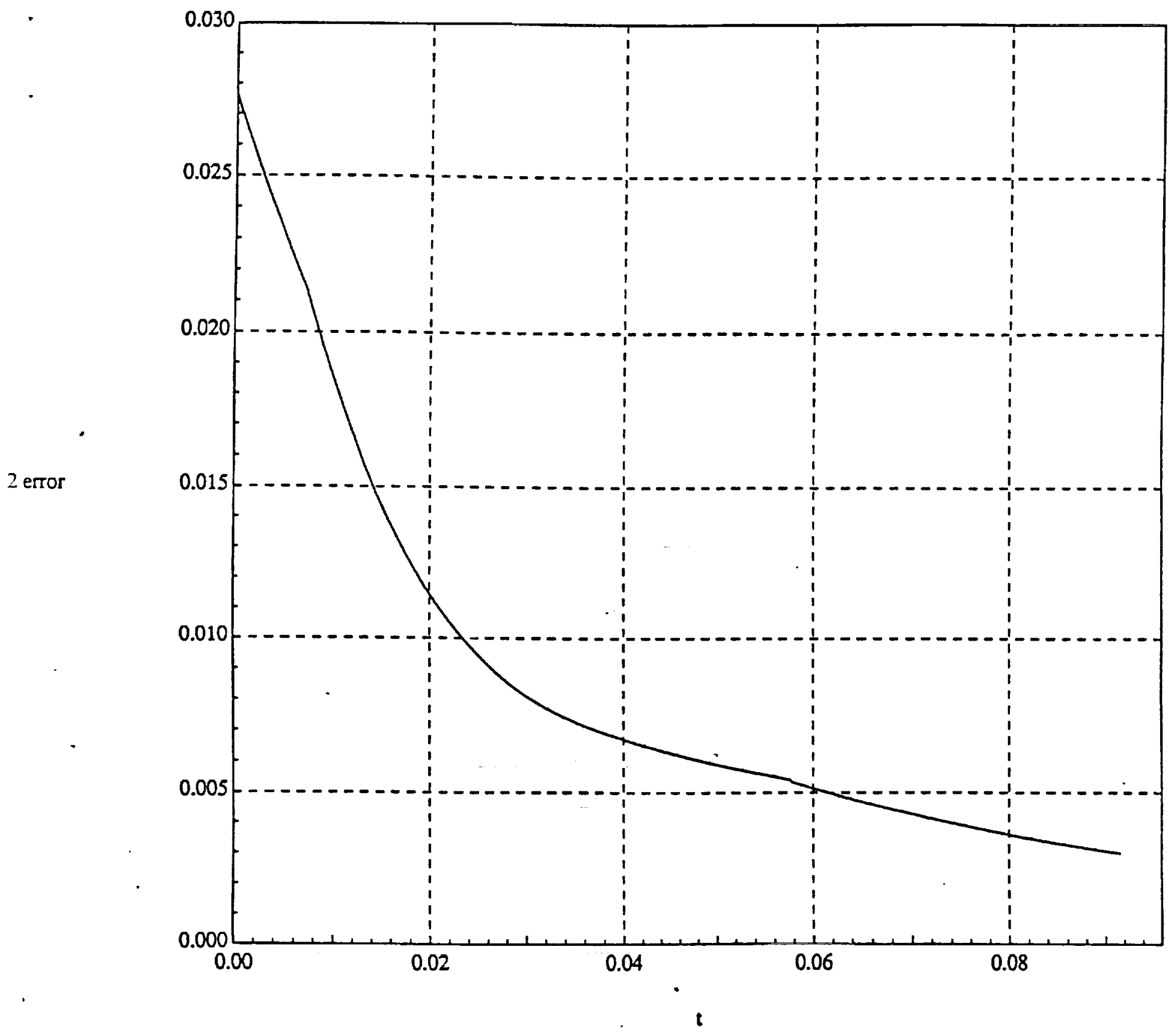

Figure 3.4. Linear Heat Equation with 4 free nodes: least squares error plotted as a function of time. 
Optimal Nodal Trajectories

Linear Heat Flow Equation

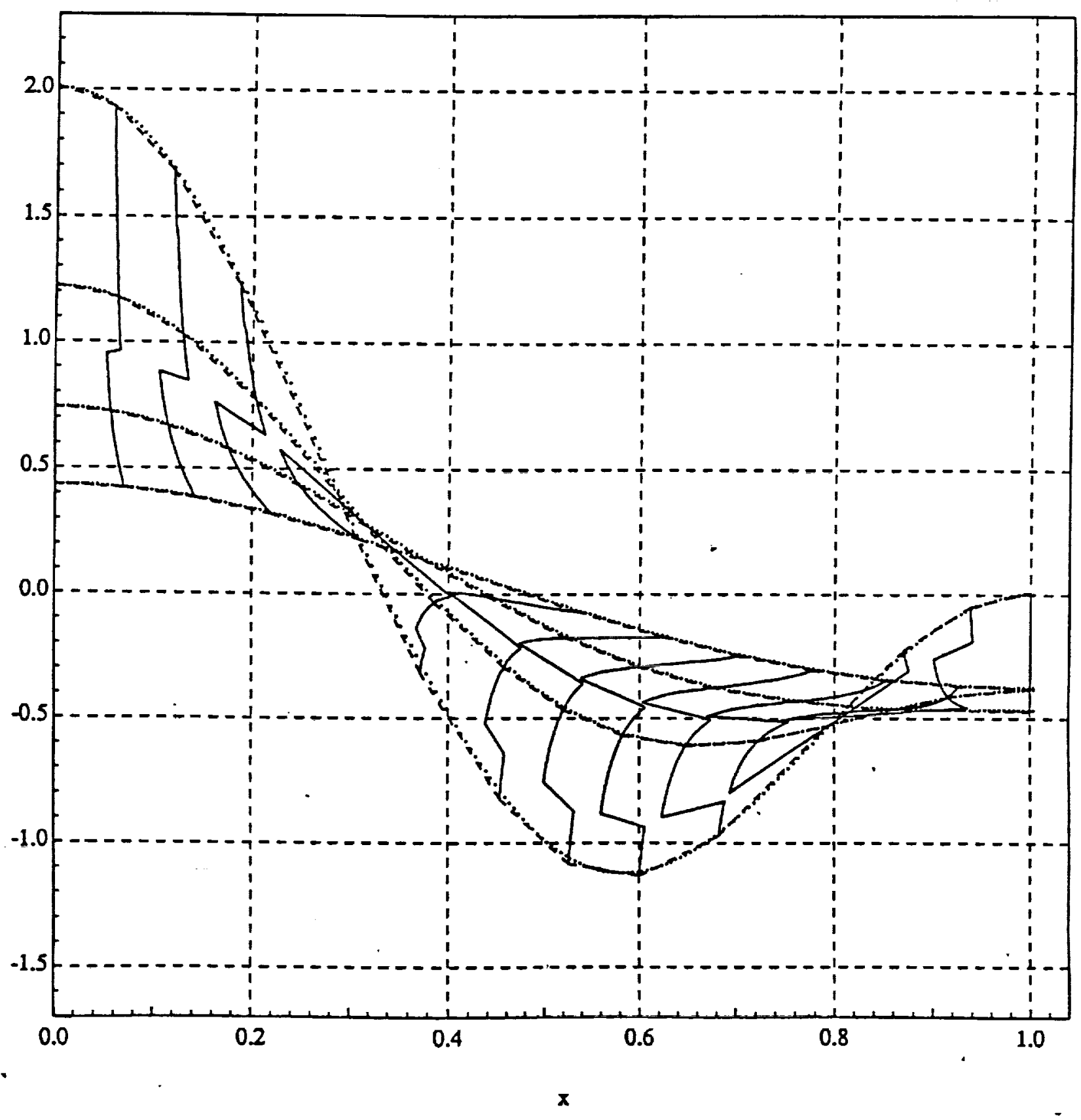

Figure 3.5. Linear Heat Equation with 10 free nodes: exact solution (dotted line), best fits (dashed line) and optimal nodal trajectories (full line) all in.u-x space. 
Optimal Nodal Trajectories

Linear Heat Flow Equation

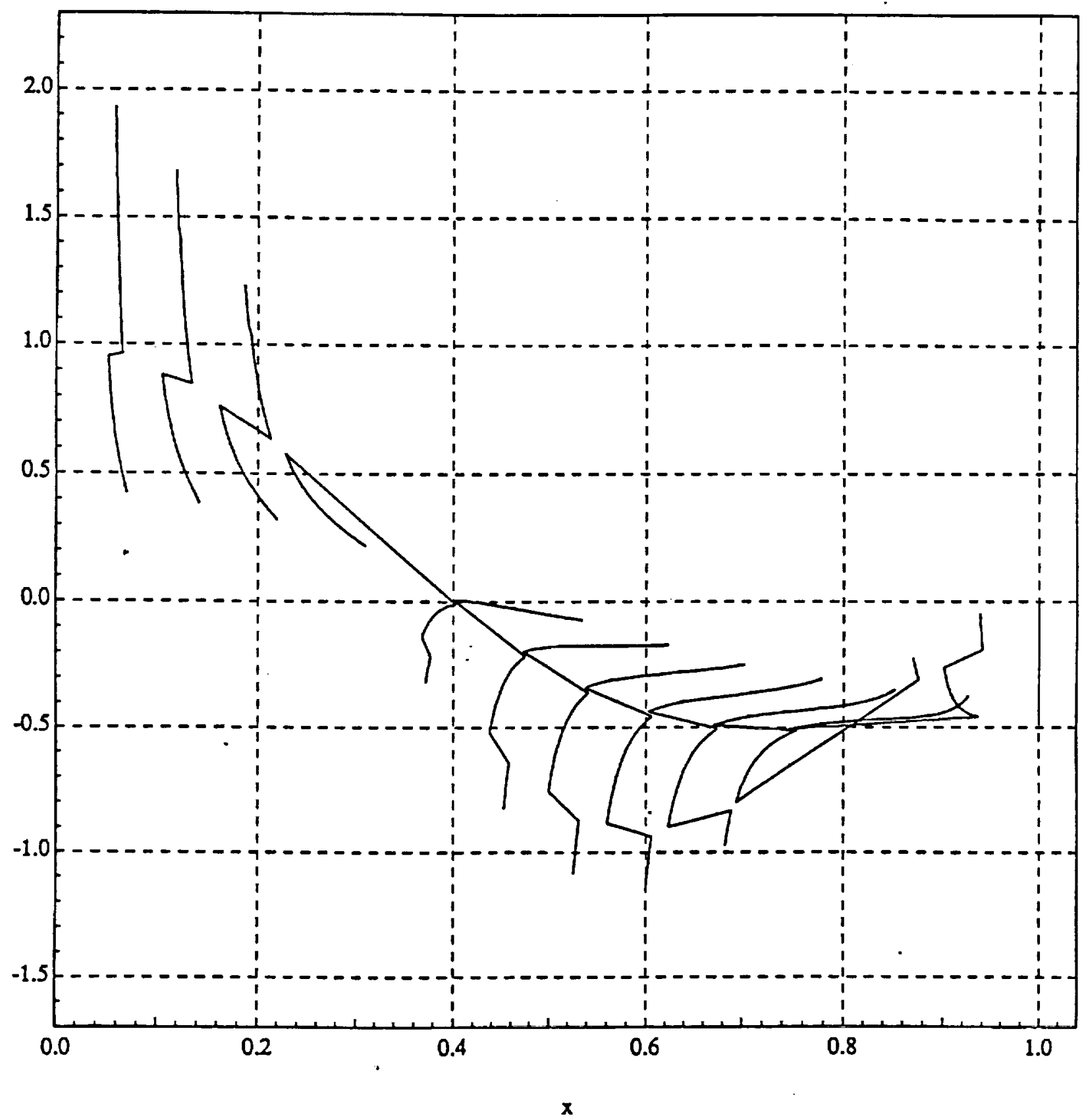

Figure 3.6. Linear Heat Equation with 10 free nodes: optimal nodal trajectories in $\mathrm{u}-\mathrm{x}$ space. 
Optimal Nodal Tsajectories in $x-t$ space

Linear Heat Flow Equation

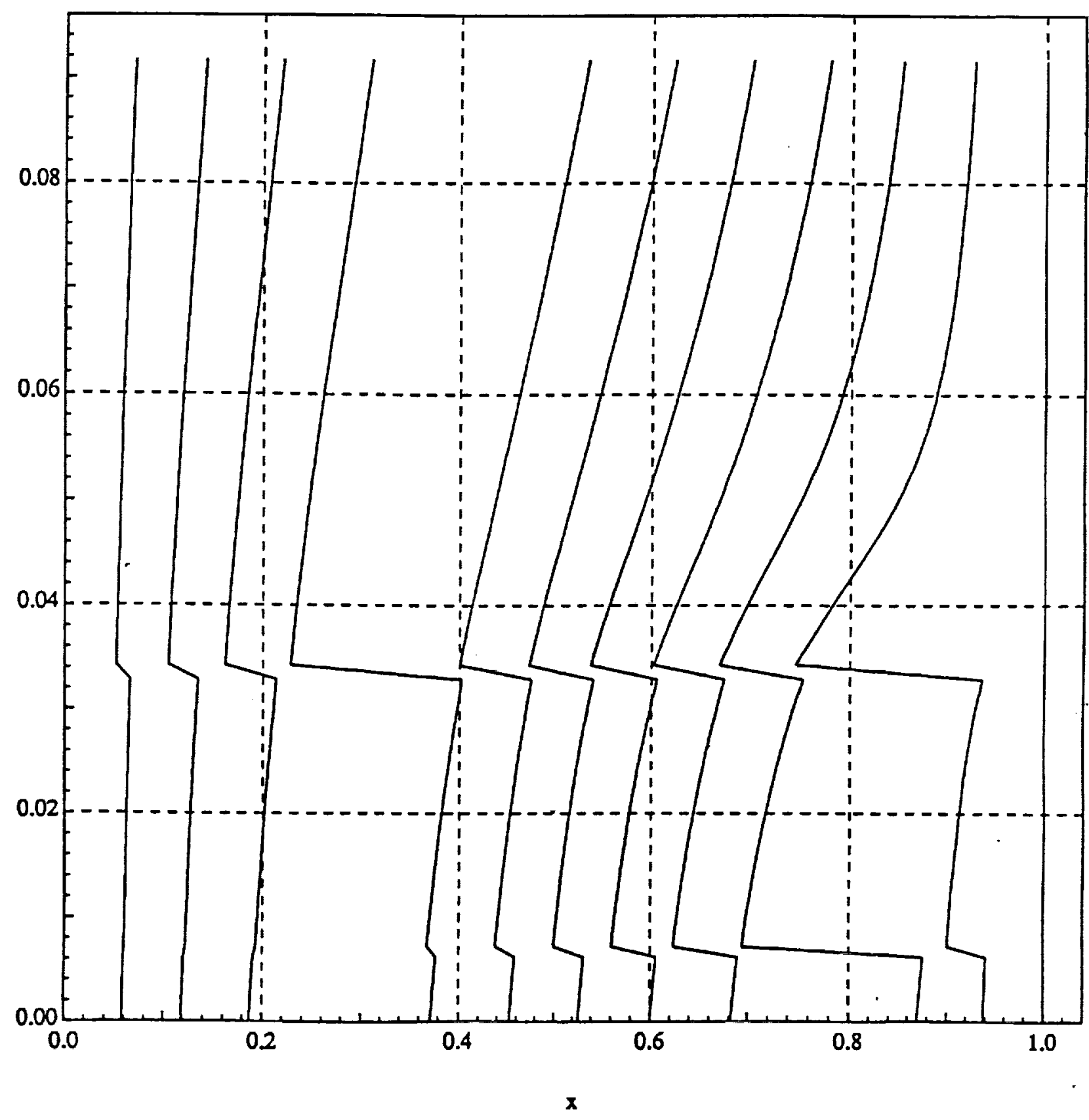

Figure 3.7. Linear Heat Equation with 10 free nodes: optimal nodal trajectories in $x$ - $t$ space. 
Least Squares Error

Linear Heat Flow Equation

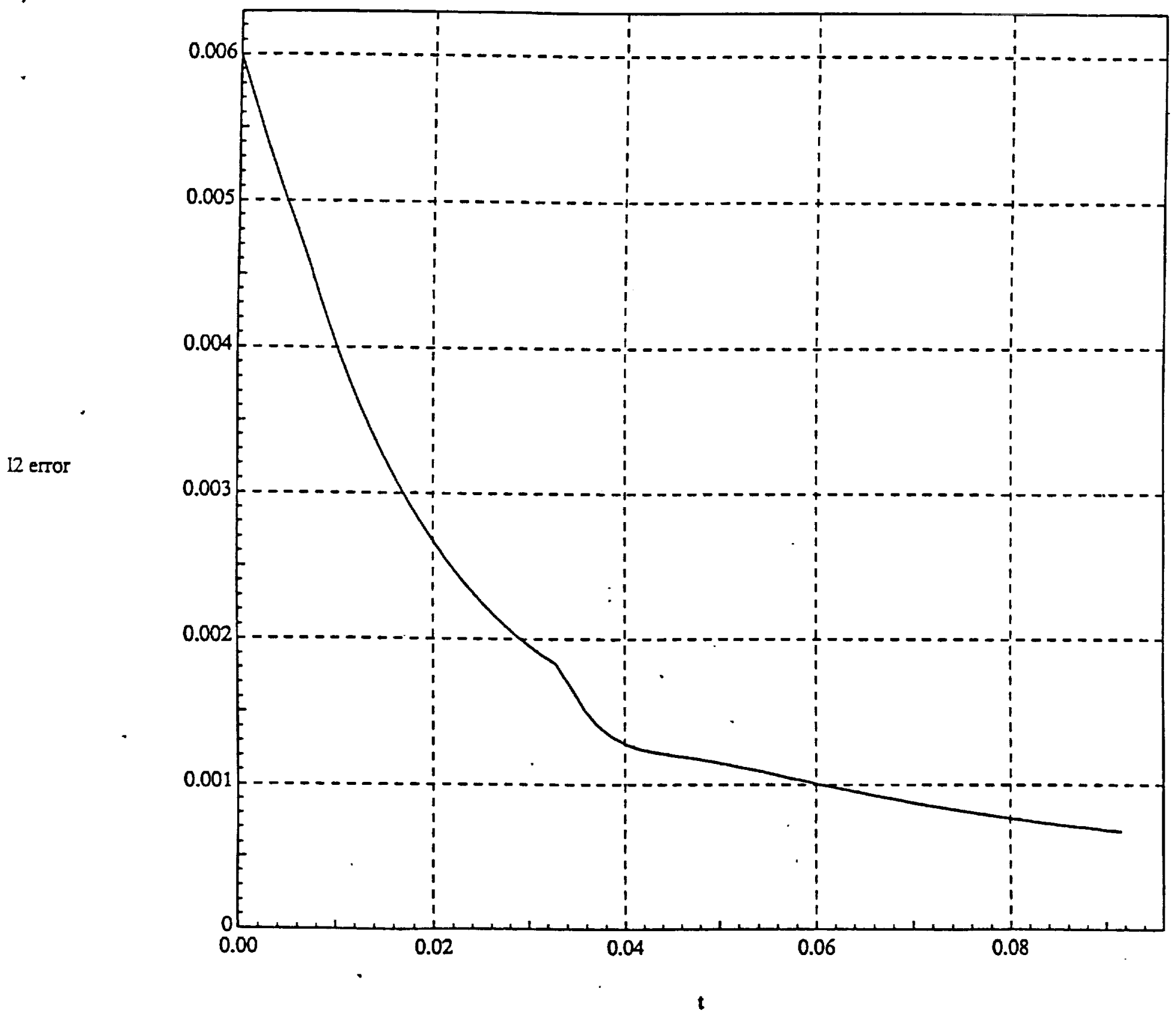

Figure 3.8. Linear Heat Equation with 10 free nodes: least squares error plotted as a function of time. 
Optimal Nodal Trajectories

Viscous Burgers Equation

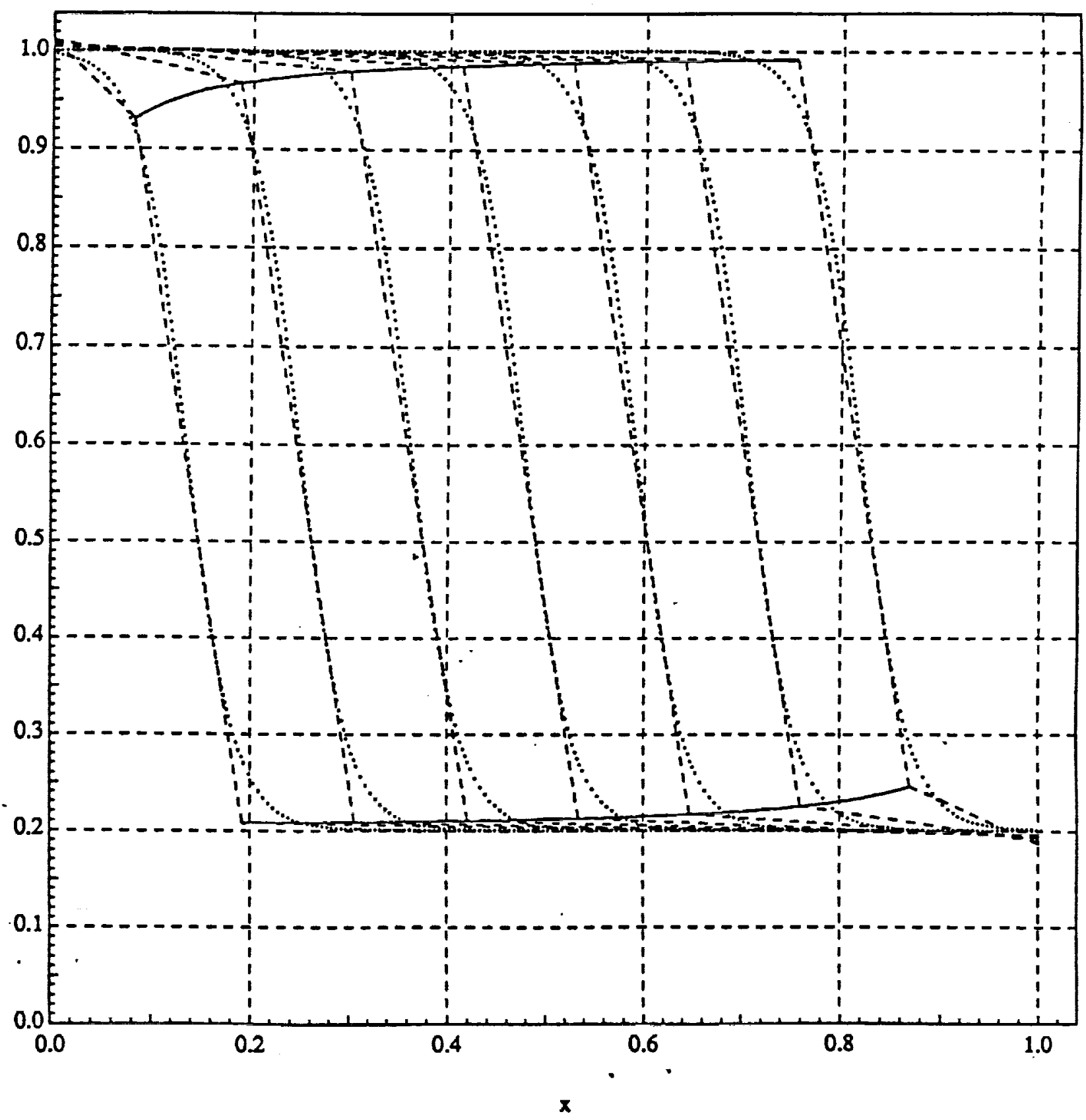

Figure 3.9. Viscous Burgers' Equation with 2 free nodes: exact solution (dotted line), best fits (dashed line) and optimal nodal trajectories (full line) all in $u-x$ space. 


\section{Optimal Nodal Trajectories}

Viscous Burgers Equation

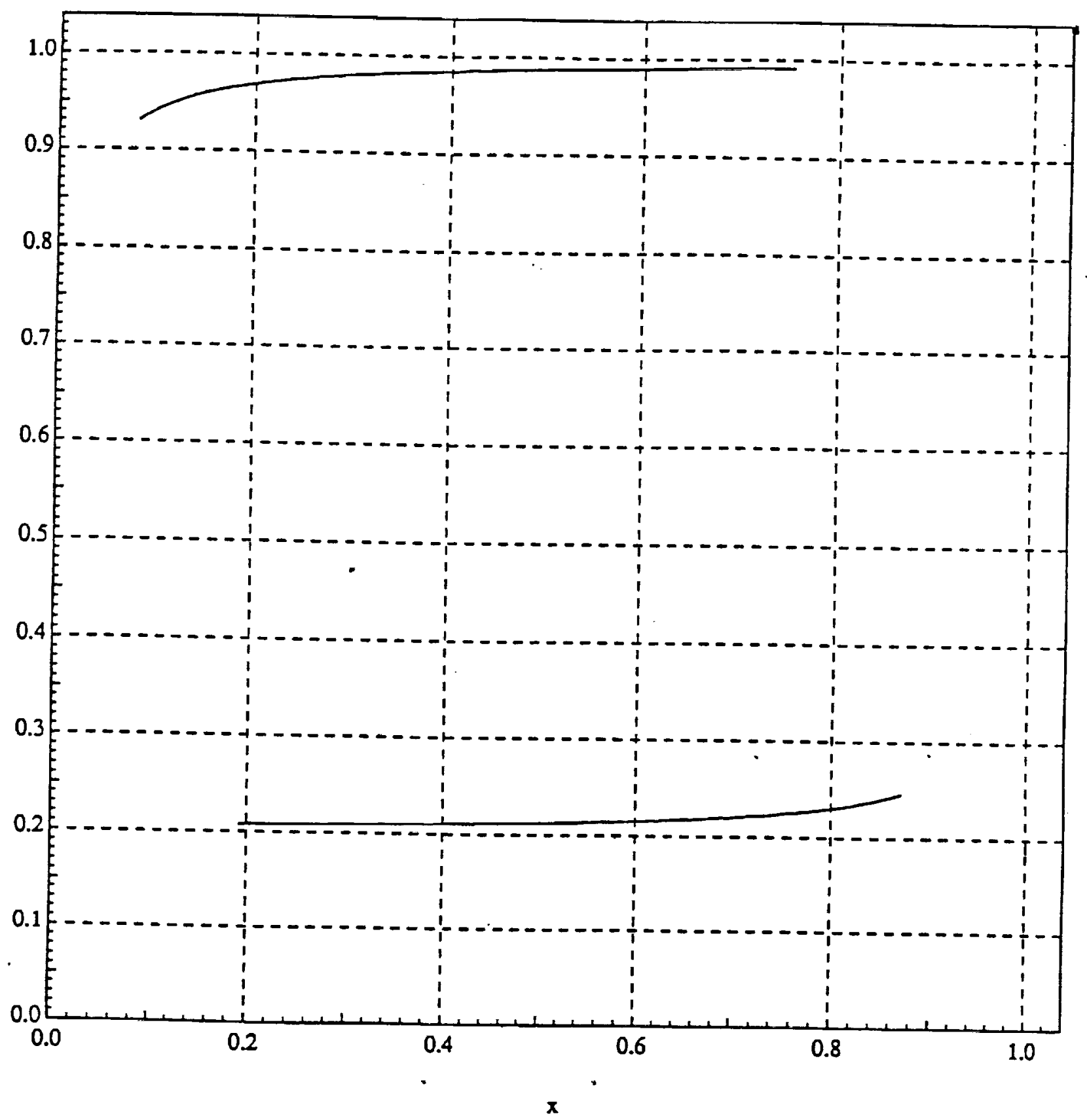

Figure 3.10. Viscous Burgers' Equation with 2 free nodes: optimal nodal trajectories in $u-x$ space. 
Optimal Nodal Trajectories in $x-t$ space

Viscous Burgers Equation

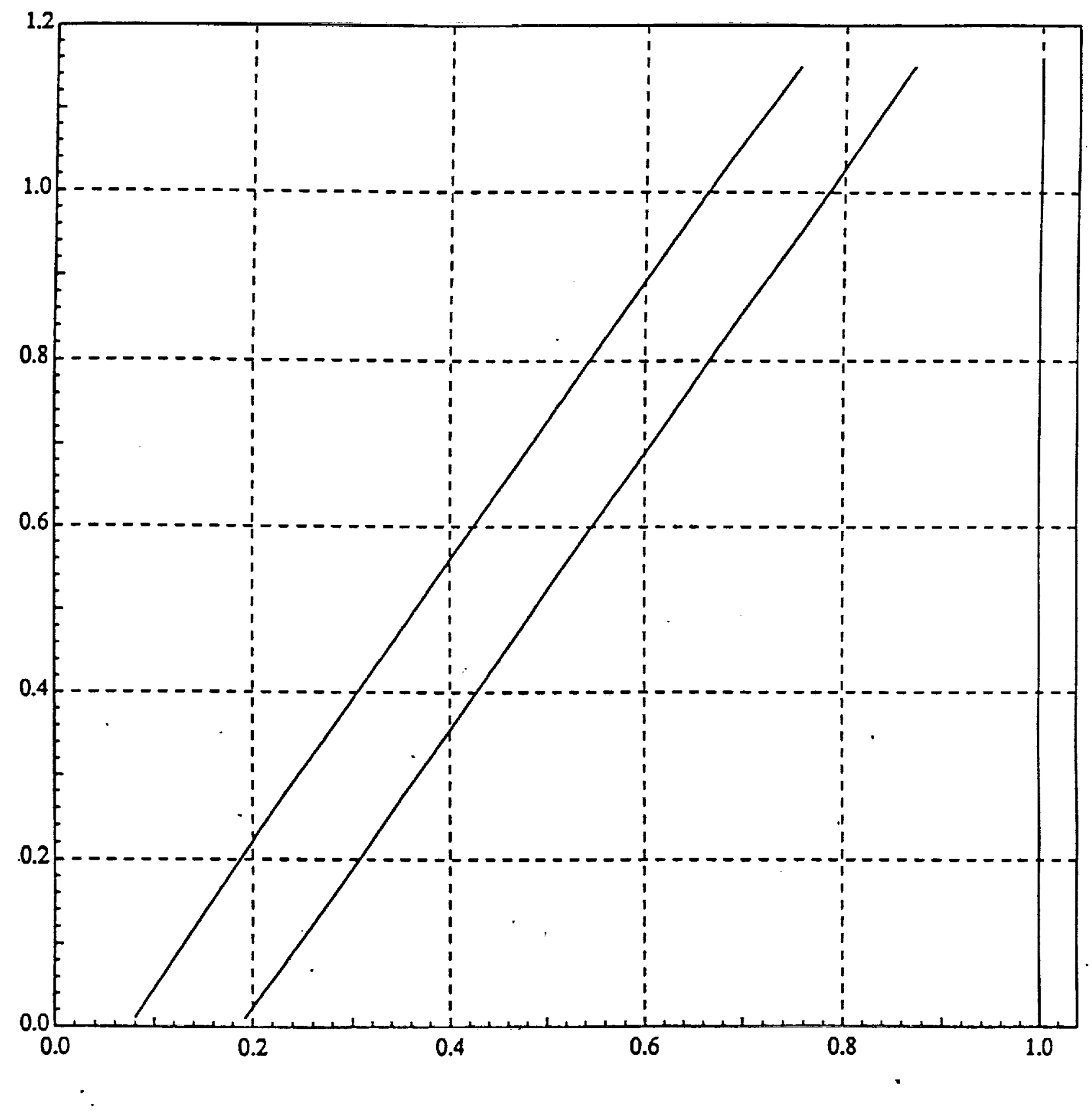

Figure 3.11. Viscous Burgers' Equation with 2 free nodes: optimal nodal trajectories in $x-t$ space. 
Least Squares Error

Viscous Burgers Equation

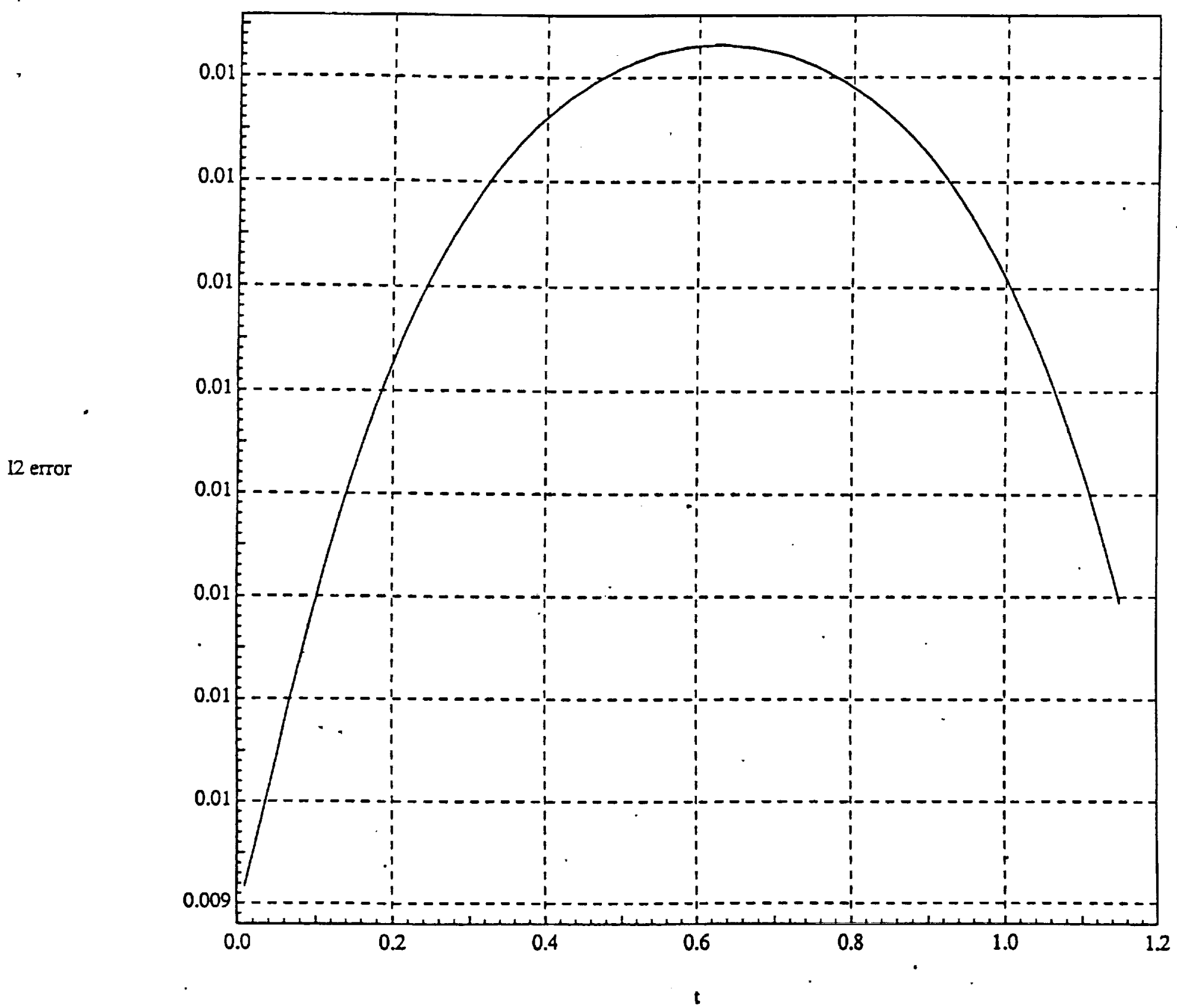

Figure 3.12. Viscous Burgers' Equation with 2 free nodes: least squares error plotted as a function of time. 
Optimal Nodal Trajectories

Viscous Burgers Equation

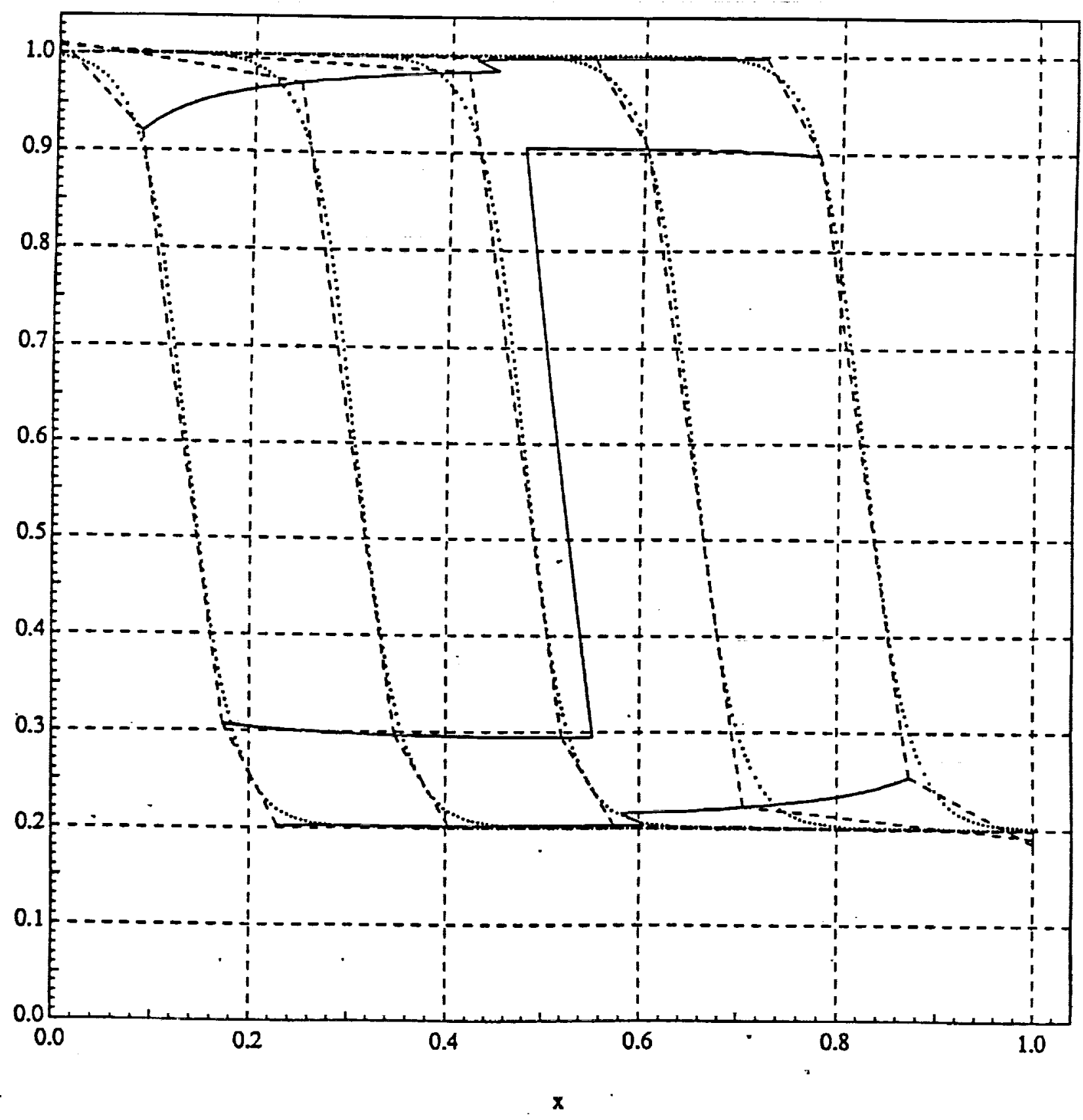

Figure 3.13. Viscous Burgers' Equation with 3 free nodes: exact solution (dotted line), best fits (dashed line) and optimal nodal trajectories (full line) all in $u-x$ space. 
Optimal Nodal Trajectories

Viscous Burgers Equation

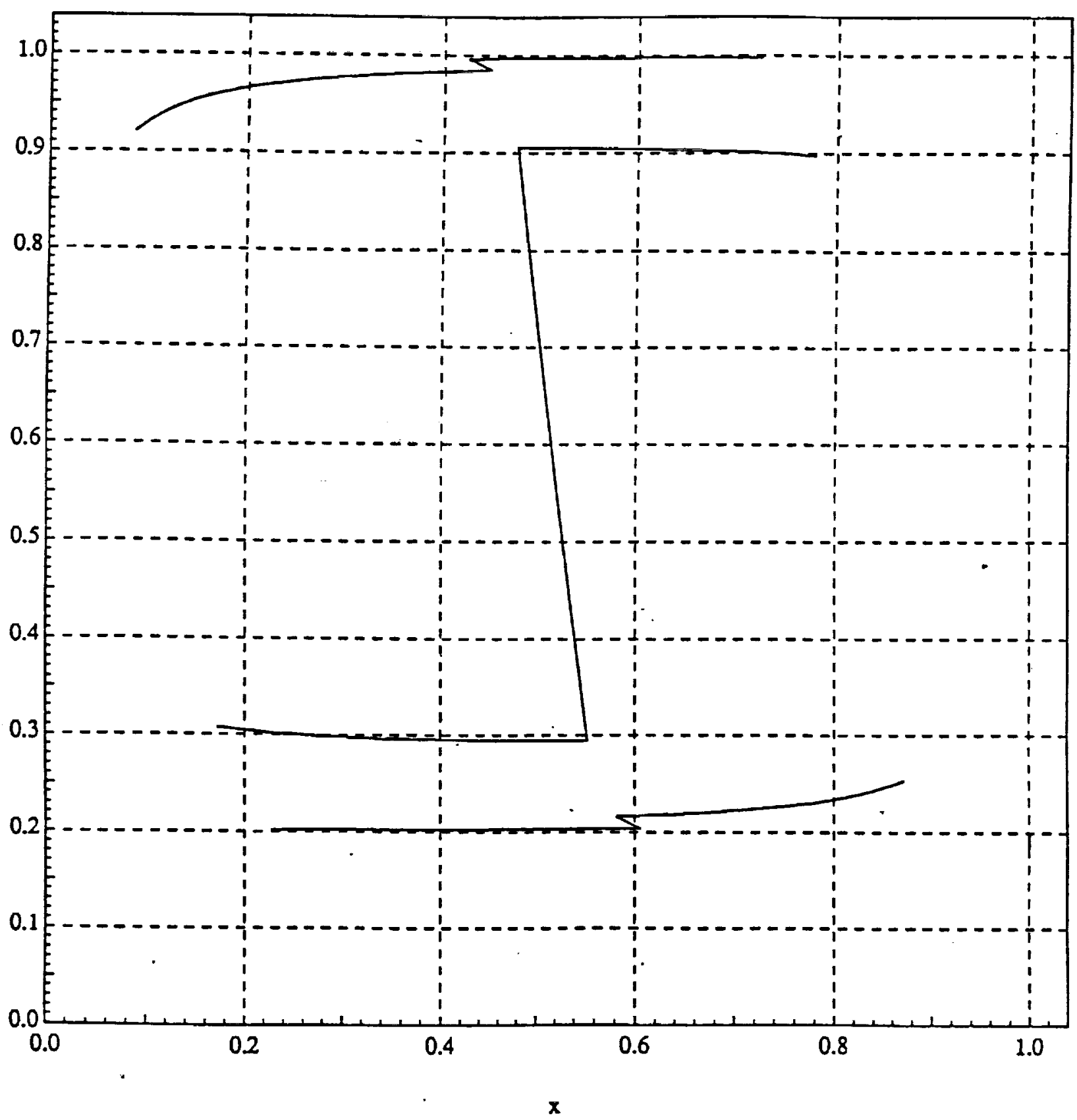

Figure 3.14. Viscous Burgers' Equation with 3 free nodes: optimal nodal trajectories in $u-x$ space. 
Optimal Nodal Trajectories in $x-t$ space Viscous Burgers Equation

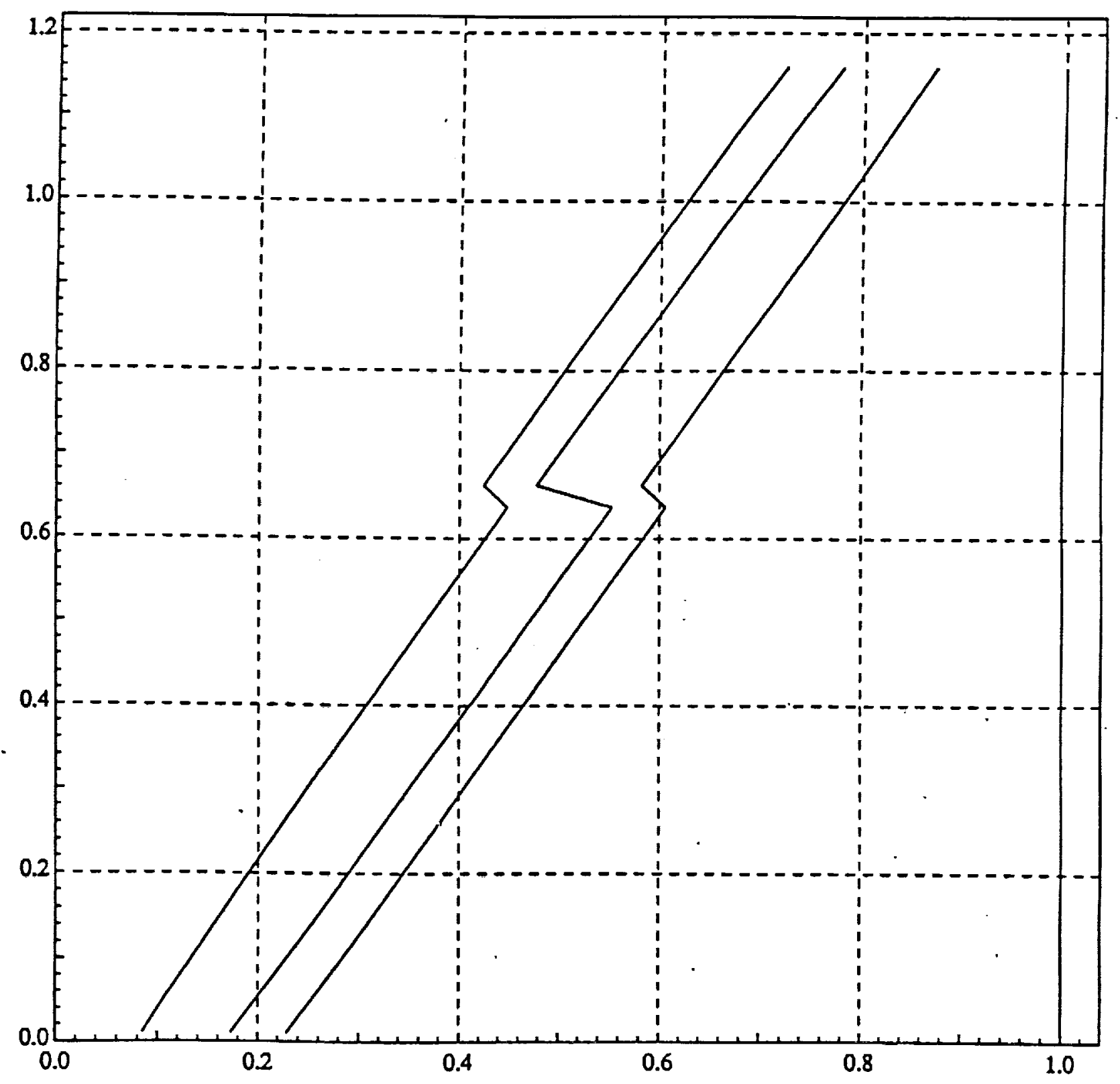

Figure 3.15. Viscous Burgers' Equation with 3 free nodes: optimal nodal trajectories in $x$-t space. 
Least Squares Error

Viscous Burgers Equation

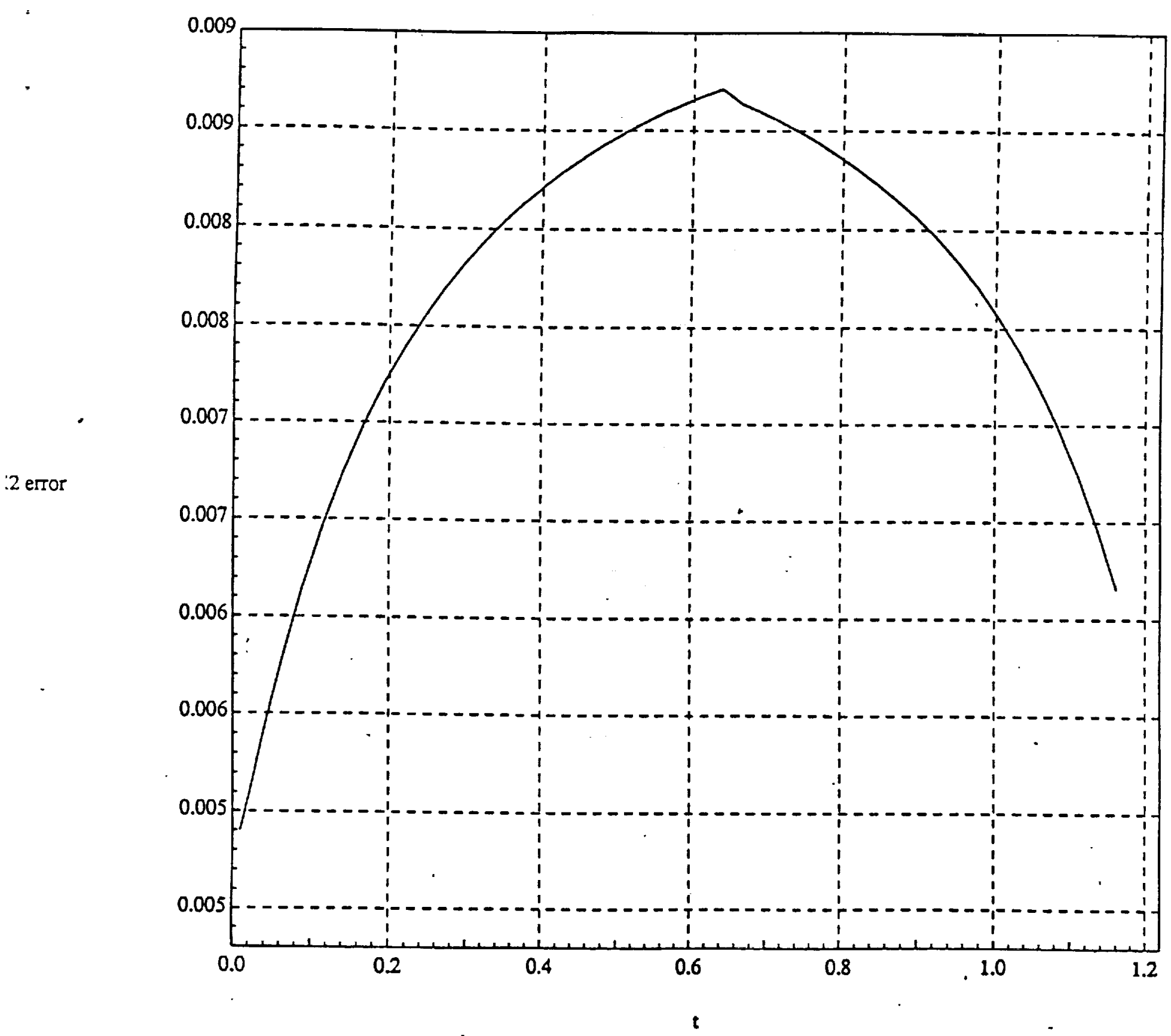

Figure 3.16. Viscous Burgers' Equation with 3 free nodes: least squares error plotted as a function of time. 
Optimal Nodal Trajectories

Viscous Burgers Equation

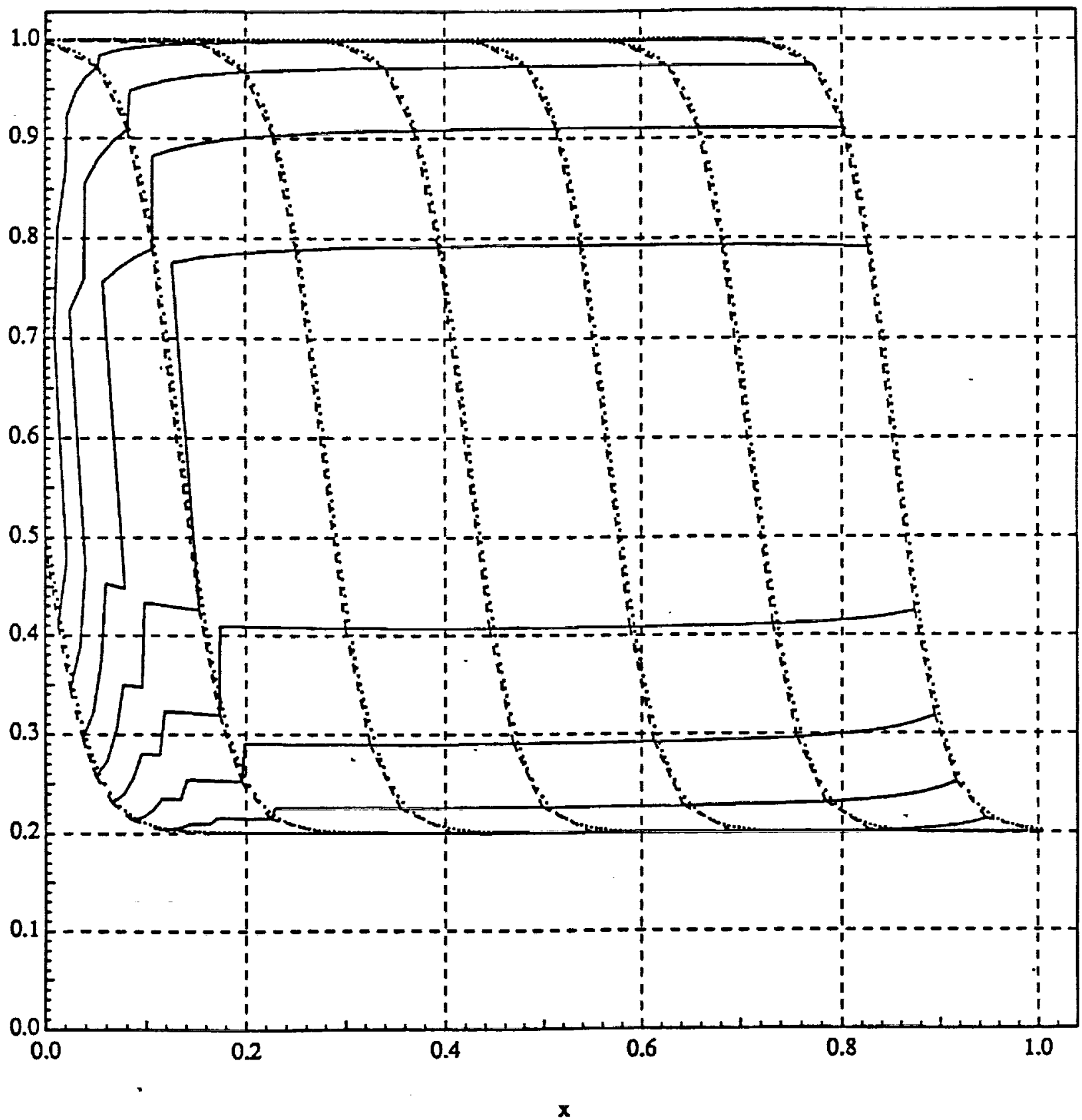

Figure 3.17. Viscous Burgers' Equation with 8 free nodes: exact solution (dotteline), best fits (dashed line) and optimal nodal trajectories (full line) all in $u-x$ space. 
Optimal Nodal Trajectories

Viscous Burgers Equation

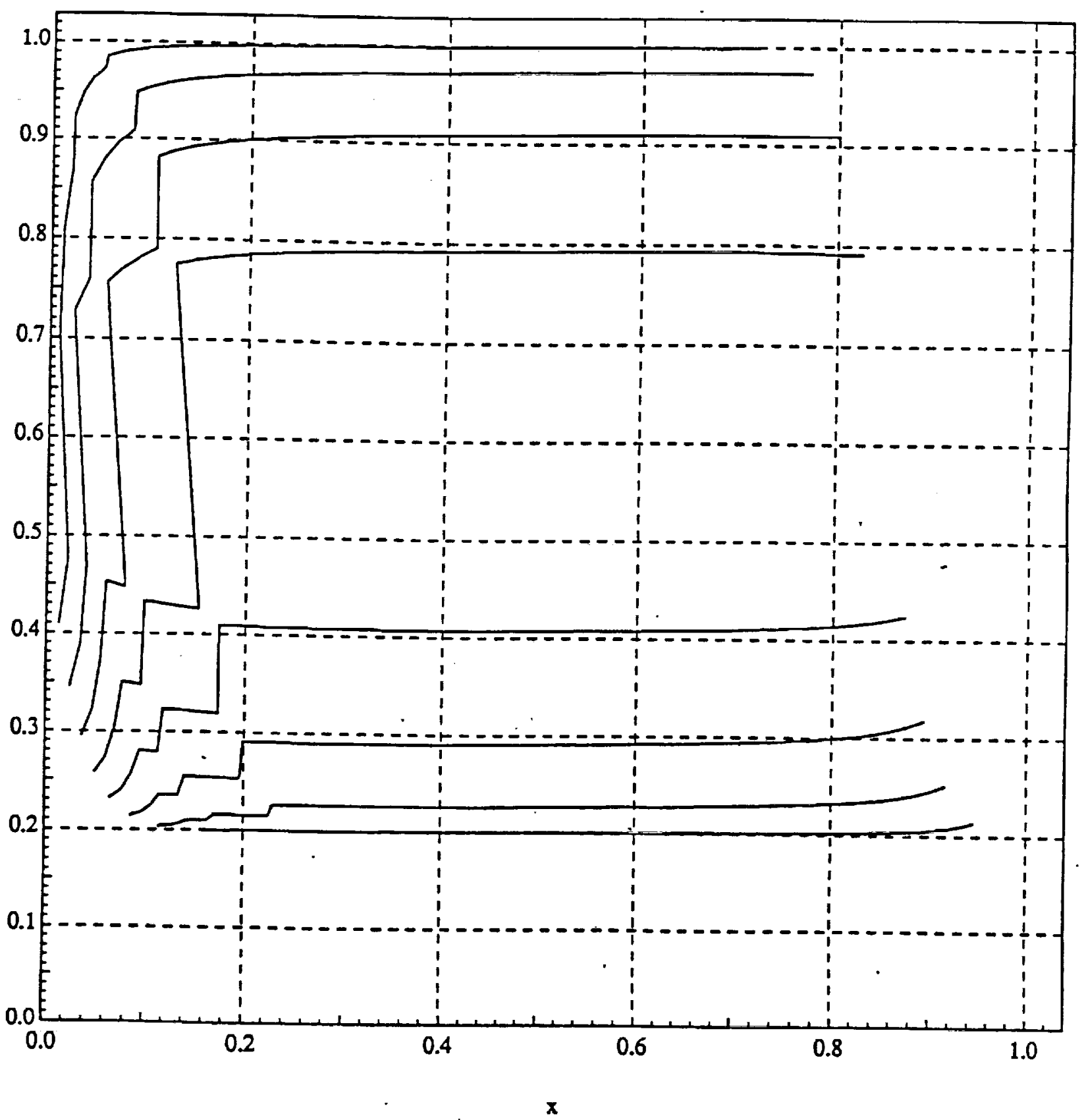

Figure 3.18. Viscous Burgers' Equation with 8 free nodes: optimal nodal trajectories in $u-x$ space. 
Optimal Nodal Trajectories in $x-t$ space Viscous Burgers Equation

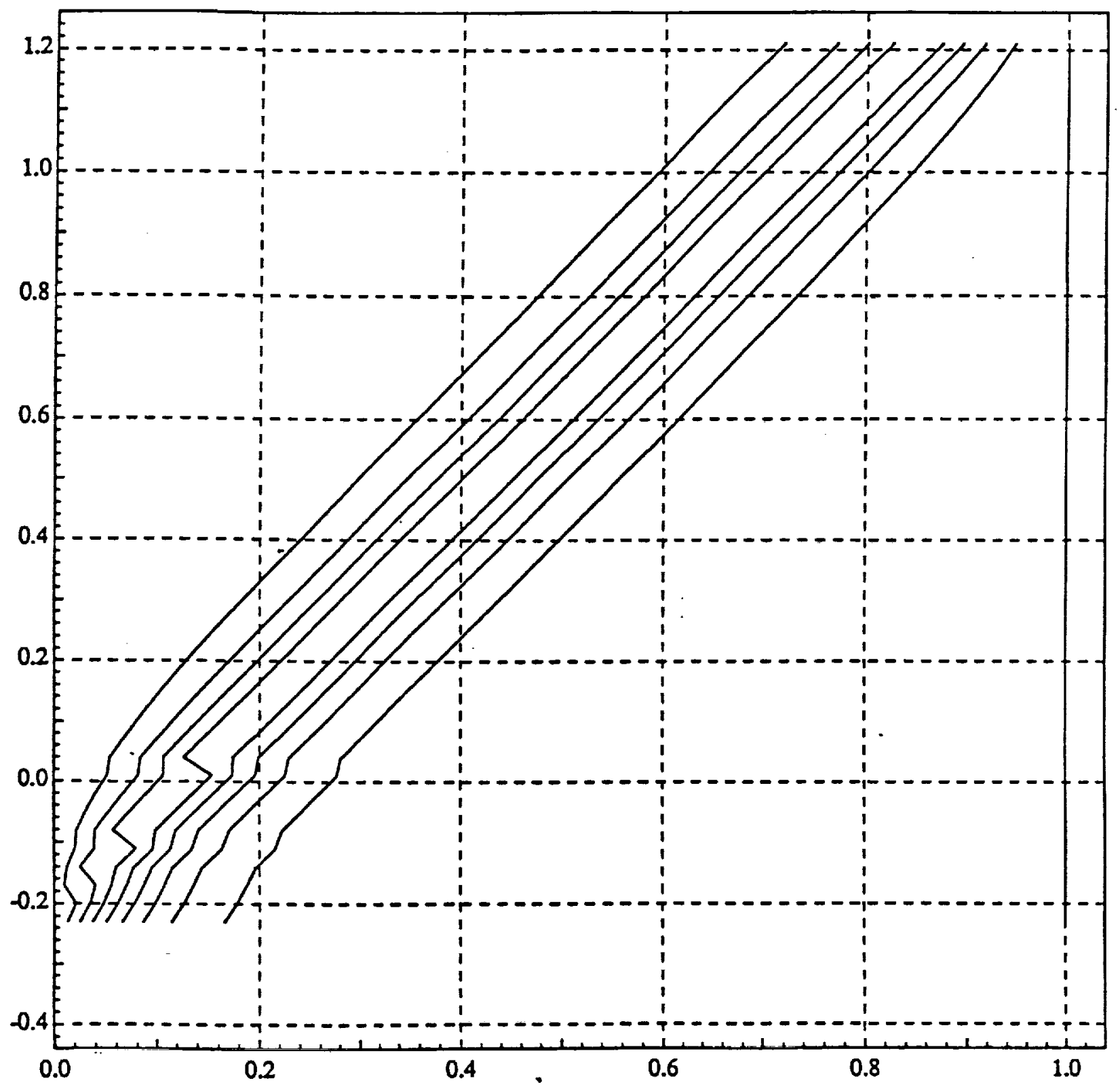

Figure 3.19. Viscous Burgers' Equation with 8 free nodes: optimal nodal trajectories in $x-t$ space. 
Least Squares Error

Viscous Burgers Equation

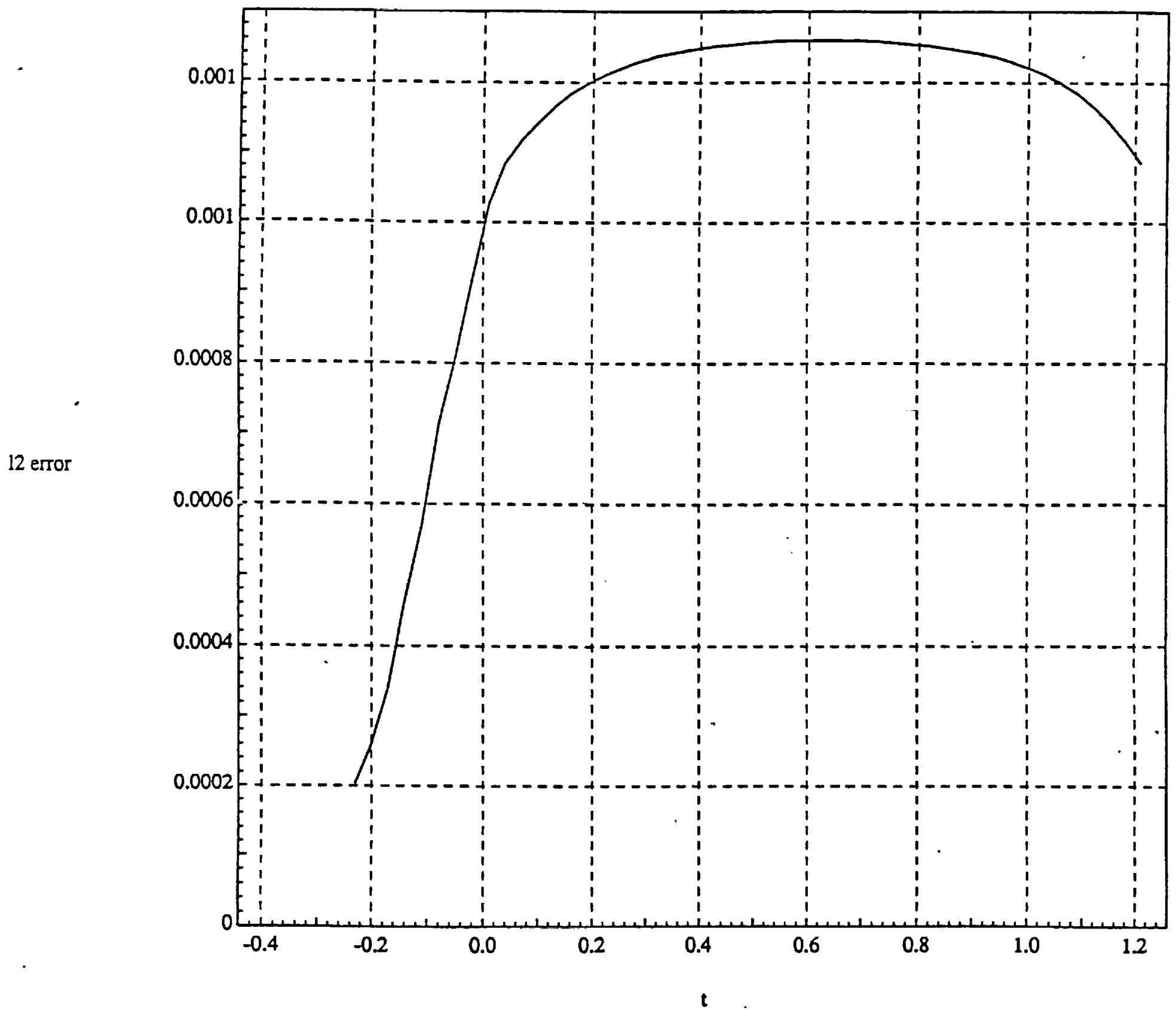

Figure 3.20. Viscous Burgers' Equation with 8 free nodes: least squares error plotted as a function of time. 


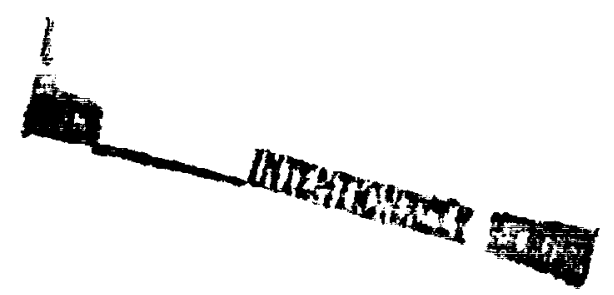

